\title{
Tumor de tritón benigno: reporte de un caso en órbita
}

\section{Orbit Benign triton Tumor: Case Report}

\author{
Fecha de recepción: 08 Diciembre 2016 | Fecha de aprobación: 31 Mayo 2017
}

\author{
TATIANA URREA-VICTORIA ${ }^{a}$ \\ Hospital Universitario San Ignacio, Colombia \\ Luis Alberto Ruiz Robles \\ Hospital Universitario San Ignacio, Colombia \\ Ana María Vanegas Monroy \\ Pontificia Universidad Javeriana, Colombia \\ Humberto Quintana Muñoz \\ Hospital Universitario San Ignacio, Colombia
}

a Autora de correspondencia. Correo electrónico: turrea@javeriana.edu.co

\section{RESUMEN}

El artículo presenta el caso de una mujer de 49 años de edad sin antecedentes patológicos con proptosis axial progresiva izquierda de un año de evolución asociada a dolor ocular, sin cambios en la agudeza visual, sin limitación en los movimientos oculares ni diplopía. Con imágenes sugestivas de seudotumor versus schwannoma versus hemangioma cavernoso orbitario versus dermoide intraconal. Fue llevada a resección de masa vía orbitotomía anterior transconjuntival. Esta fue una lesión compatible histológicamente con hamartoma neuromuscular (tumor de tritón benigno).

Palabras clave

neoplasia; órbita; tumor de tritón; hamartoma; neuromuscular; coristoma; tumor benigno de tritón.

Cómo citar: Urrea-Victoria T, Ruiz Robles LA, Vanegas Monroy AM, Quintana Muñoz H. Tumor de tritón benigno: reporte de un caso en órbita. Univ Med. 2017;58(4):1-5. doi: http://dx.doi.org/10.11144/Jav eriana.umed58-4.temm

\begin{abstract}
This article shows the case of a 49-year-old woman with no medical history, developed a progressive left axial proptosis associated with ocular pain. There are no changes to visual acuity, limitation of eye movement or diplopia. The diagnostic images may suggest one of the following: pseudotumor, schwannoma, orbital cavernous hemangioma or intraconal dermoid. The resected specimen was taken via anterior transconjuntival orbitotomy. Microscopic examination of the tumor showed a neuromuscular hamartoma (benign triton tumor).

Keywords

Neoplasm; orbit; tumor (triton); neuromuscular; hamartoma; orbit; choristoma;
\end{abstract} benign triton tumor.

\section{Introducción}

Los tumores compuestos por músculo esquelético y elementos neurales se conocen colectivamente como tumores tritón $[1,2]$. Su nombre se ha derivado a partir del anfibio (salamandra), en el que el nervio normal parece inducir 
la regeneración de músculo esquelético $[1,2$, $3]$. Es un tumor muy raro que consta de fibras nerviosas maduras y fibras musculares estriadas. La mayoría de los casos han sido reportados en asociación con grandes nervios y se describe en los lactantes y niños pequeños $[1,2,4]$.

Hay solo cinco casos con tumor benigno de tritón reportados en los nervios periféricos de la cabeza y el cuello [1, 2, 3]. En la literatura sobre el tema se ha reportado una leve predominancia por el sexo femenino 2,4:1, en comparación con los hombres [2]. En esta ocasión se presenta un caso de un tumor de tritón que se desarrolló en la región intraorbitaria de una mujer adulta.

El mejor reconocido de estos tumores es el tumor rabdomioblástico (tumor maligno de tritón) [5], aunque también se producen combinaciones como rabdomiosarcoma con las células ganglionares (ectomesenquimoma). Las lesiones benignas compuestas de diferenciación neuronal y muscular esquelética son raras y están representadas principalmente por el hamartoma neuromuscular o coristoma y el neurofibroma, con un componente rabdomiomatoso [5].

Aún no está del todo claro la patogénesis de estos tumores. Se cree que son lesiones hamartomatosas de origen neuroectodérmico mesenquimal que podrían deberse a la incorporación de tejido mesenquimal en las vainas nerviosas durante la embriogénesis o a una diferenciación aberrante de elementos neuroectodérmicos en componentes mesenquimales o por crecimiento hamartomatoso de los husos musculares [3].

Macroscópicamente, son masas multinodulares subdivididas por bandas fibrosas en nódulos pequeños o fascículos [5]. Histopatológicamente, cada fascículo se compone de fibras musculares esqueléticas altamente diferenciadas que varían en tamaño, pero a menudo son más grandes de lo normal [4]. Los fascículos se componen de fibras de músculo estriado maduros íntimamente asociados con las fibras nerviosas y encerrados dentro de la misma vaina fibrosa perimisial, separados por colágeno maduro en nódulos y fascículos más pequeños. A veces, el componente fibroso que rodea las lesiones es tan denso y celular que sugiere el diagnóstico de un tumor fibromatoso [3], lo que hace el diagnóstico más difícil.

Las tinciones de inmunohistoquímica para los marcadores neurales y musculares resaltan la estrecha yuxtaposición de ambos componentes [5]. En este artículo reportamos el caso, debido a la rareza del tumor de tritón, sobre todo en edad adulta, y aún más en la región orbitaria.

\section{Presentación del caso}

El caso corresponde a una mujer de 49 años de edad, sin antecedentes patológicos, valorada por el servicio de oftalmología oncológica y oculoplastia en el Hospital Universitario San Ignacio. La paciente consultó por un cuadro de proptosis axial progresiva izquierda de un año de evolución asociada a dolor ocular sin cambios en la agudeza visual, sin limitación en los movimientos oculares ni diplopía.

En el examen oftalmológico presentaba agudeza visual lejana sin corrección de 20/20 en ambos ojos, sin alteración pupilar. El test de Ishihara fue normal. No tenía limitación de los movimientos oculares, ni alteración en los segmentos anterior ni posterior; únicamente se evidenciaba proptosis axial izquierda con diferencia de $4 \mathrm{~mm}$ con respecto al ojo contralateral (exoftalmometría base: $100 \mathrm{~mm}$; ojo derecho: $17 \mathrm{~mm}$; ojo izquierdo: $21 \mathrm{~mm}$ ).

Se le tomaron las siguientes imágenes diagnósticas: tomografía axial computarizada (TAC) de órbitas (figuras la y 1b), resonancia magnética nuclear (RMN) (figuras 2a, 2b, 2c y 2d) y ecografía de órbitas.

Figura 1.TAC de órbitas: lesión de $16 \times 12 \times 8 \mathrm{~mm}$ con densidad de tejidos blandos, de localización intraconal en órbita izquierda que está en contacto con el nervio óptico. Se consideraron posibilidades diagnósticas: seudotumor vs. schwannoma

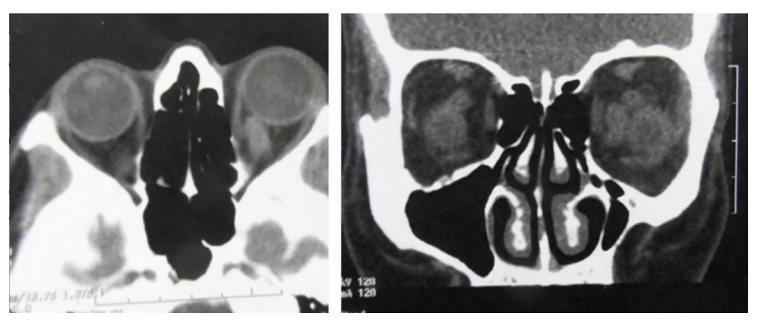


Figura 2.RMN de órbitas. Lesión orbitaria intraconal medial en íntimo contacto con el recto inferior y recto medio. Rechaza lateral al nervio óptico. Su aspecto es ovalado y con aparentes contornos definidos. Sugieren diagnóstico de hemangioma cavernoso orbitario izquierdo
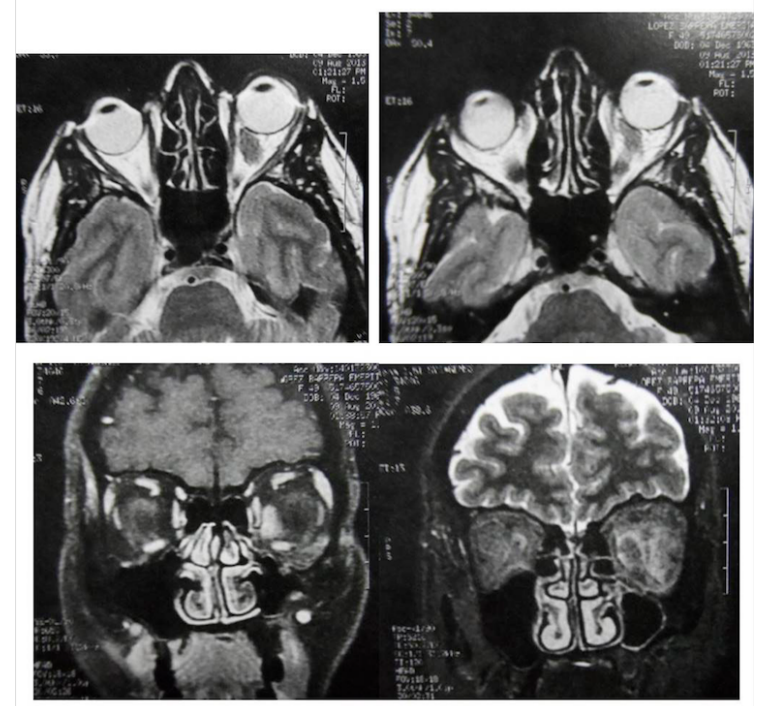

En la ecografía de órbita se encontró una masa orbitaria compatible con quiste (entre el nervio óptico y el músculo recto medio en el tercio profundo de la órbita).

Dados los hallazgos imaginológicos, se consideró una lesión sugestiva de hemangioma cavernoso como primera posibilidad. Así, teniendo en cuenta la localización, se decidió, en junta con el servicio de otorrinolaringología y luego de valoración por el servicio de neurocirugía, la resección de la masa por abordaje transcraneano.

Así mismo, por la ausencia de afectación neurológica, en la junta quirúrgica se consideraron dos posibles opciones de abordaje quirúrgico: transnasal-transesfenoidal extendido inferomedial $\mathrm{u}$ orbitotomía anterior transconjuntival más crioterapia.

La paciente fue llevada a resección de la masa vía orbitortomía anterior transconjuntival. El hallazgo intraquirúrgico fue de una masa adyacente al polo posterior, entre el borde inferior del recto medial y el borde medial del recto inferior, de aspecto friable, violácea, aparentemente vascular, firmemente adherida, sin planos de clivaje claros de aproximadamente
$10 \mathrm{~mm}$ (figura 3). Hubo una resección incompleta, debido a la ubicación y por el elevado riesgo de generar una lesión visual y neurológica.

Figura 3.Pieza quirúrgica: masa intraorbitaria

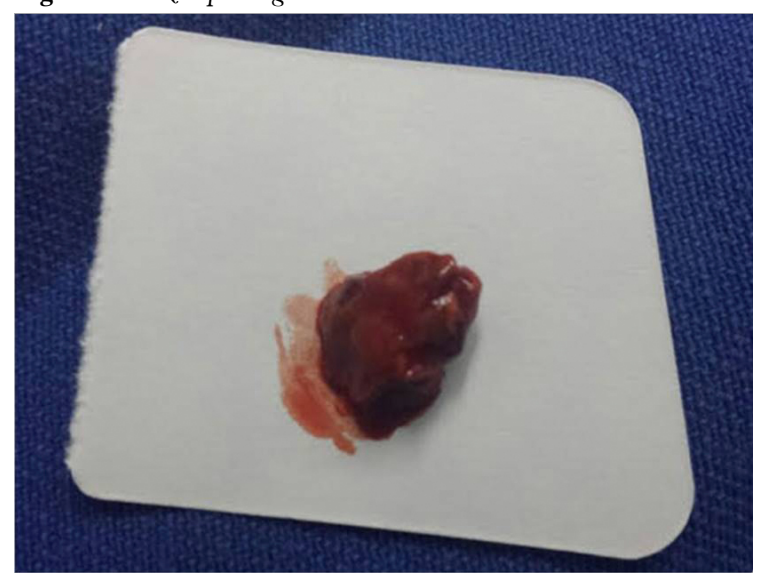

Reporte de patología

En las figuras $4 a, 4 b$ y $4 c$ se muestra el reporte del servicio de patología. En la descripción microscópica, los cortes evidencian una lesión benigna constituida por haces gruesos de tejido neural entremezclados con músculo estriado y tejido fibroadiposo maduro.

Figura 4a.Coloración de hematoxilina y eosina, donde se identifican haces de músculo estriado y neural

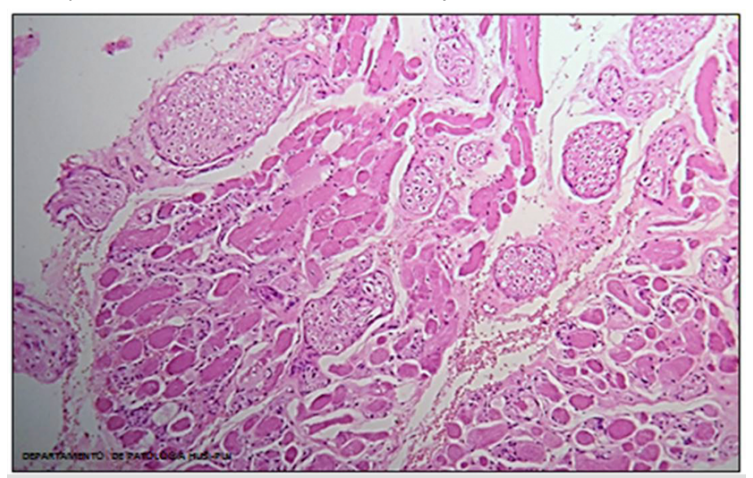


Tatiana Urrea-Victoria, Luis Alberto Ruiz Robles, Ana María Vanegas Monroy et al.

Figura 4b.Hematoxilina y eosina. Se observa cómo los haces de tejido nervioso disecan los haces musculares

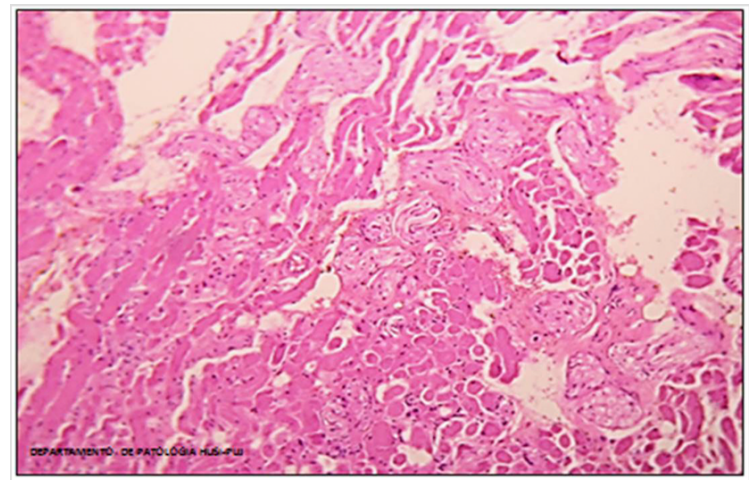

Figura 4c.Hematoxilina y eosina a 40X

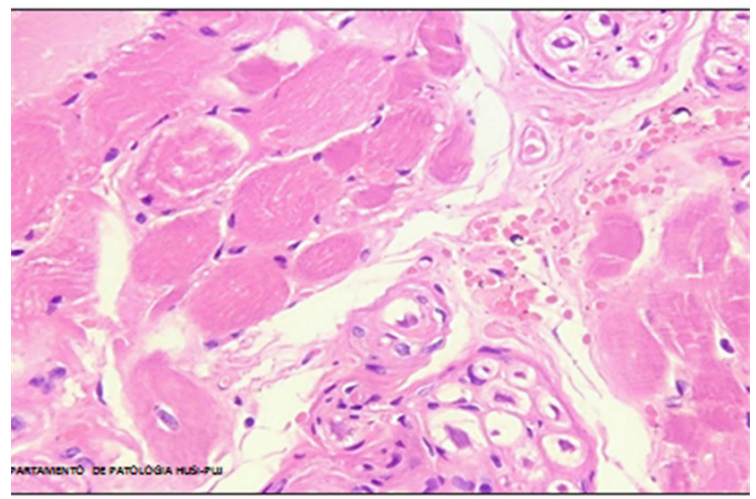

Luego de los análisis se diagnosticó una lesión compatible histológicamente con hamartoma neuromuscular (tumor de tritón benigno). La paciente evolucionó con preservación de la agudeza visual, sin restricción de la motilidad y con discreta diplopía vertical, manejada con ejercicios y oclusión por ortóptica (figuras 5 a y $5 b)$.

Figura 5a.Se evidencian movimientos oculares sin restricción. No hay limitación para la aducción del ojo izquierdo

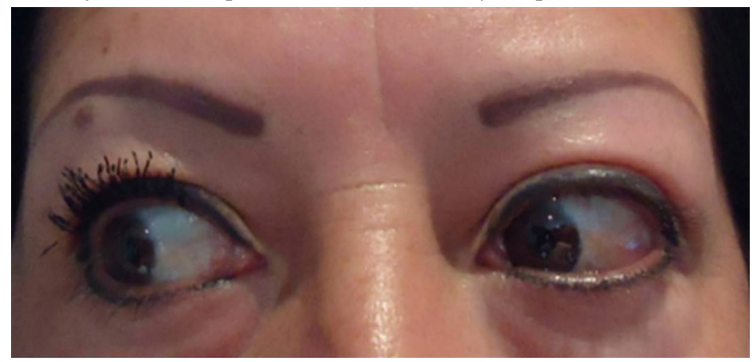

Figura 5b.Vista frontal

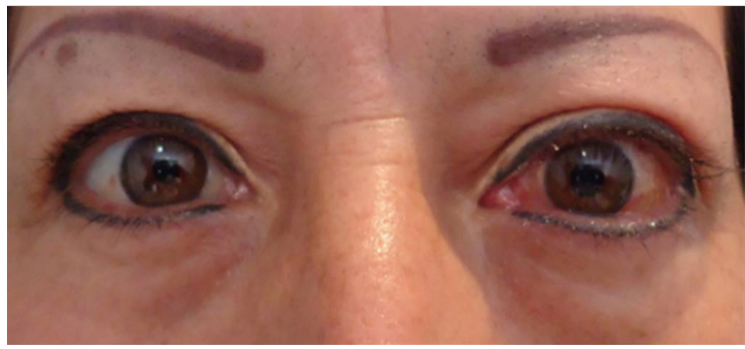

A la paciente se le realizó control imaginológico a los dos meses del postoperatorio (figuras 6a y 6b). Este reportó una masa residual $(10 \mathrm{~mm} \times 6 \mathrm{~mm})$ en estrecho contacto con el músculo recto medial y con el nervio óptico que, comparada con imágenes previas, evidenció una notoria reducción de su tamaño. Se continuaron los controles oftalmológicos periódicos que evidenciaron disminución de la proptosis y la diplopía y agudeza visual conservada.

Figura 6 (a y b).TAC de cráneo. Cortes sagital y coronal. Se evidencia masa residual en estrecho contacto con el músculo recto medial y con el nervio óptico en el lado izquierdo

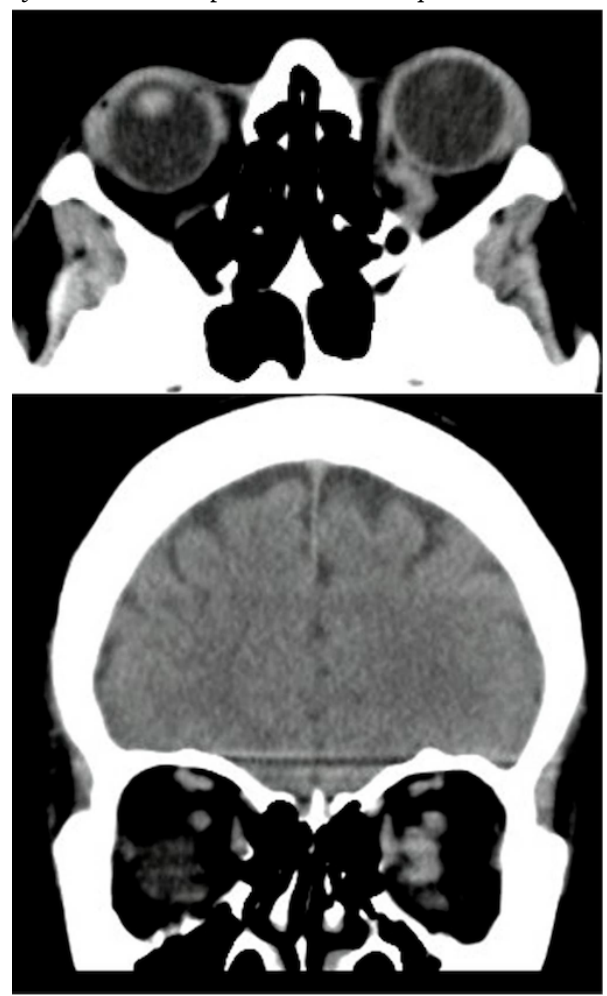




\section{Conclusiones}

El diagnóstico de un tumor benigno de tritón depende únicamente del examen histopatológico, ya que es difícil la sospecha y el diagnóstico clínico [3]. El tratamiento debe ser conservador y estar dirigido, principalmente, a mantener la integridad del nervio óptico. Se ha establecido que, aun cuando la escisión completa es totalmente curativa [3], el pronóstico -en la mayoría de los casos - es muy bueno, pese a que se han reportado algunas recurrencias [2]. En nuestro caso, teniendo en cuenta la proximidad al nervio óptico y las adherencias a sus envolturas, se decidió no reintervenir quirúrgicamente, dado el hallazgo benigno histológico en la patología.

\section{Referencias}

1. Amita K, Shankar SV. Benign tritón tumor: a rare entity in head and neck region. Korean J Pathol. 2013;47:74-6.

2. Bae DH, Kim $\mathrm{CH}$, Cheong JH, Kim JM. Adulthood benign tritón tumor developed in the orbit. J Korean Neurosurg Soc. 2014;56(2):146-8.

3. Hemalatha AL, Sanjay M, Anoosha K, Ashok KP, Shantha Kumari BR. Benign tritón tumour of upper lipa rare neoplasm at an extremely uncommon site. J Clin Diagnostic Res [Internet]. 2014;8(12):FD03-4. doi: 10.7860/JCDR/2014/10289.52955

4. Thakrar R, Robson CD, Vargas SO, Meara JG, Rahbar R, Smith ER. Benign tritón tumor: multidisciplinary approach to diagnosis and treatment. Pediatr Dev Pathol [Internet]. 2014;17(5):400-5. Disponible en: http://www.pedpath.org/doi /abs/10.2350/14-04-1459-CR.1

5. Dodge HW, Craig WM. Benign tumors of peripheral nerves and their masquerade. Minn Med. 1957;40(5):294-301. 